\title{
Impact on Sea Surface Salinity Retrieval of Different Auxiliary Data Within the SMOS Mission
}

\author{
Roberto Sabia, Student Member, IEEE, Adriano Camps, Senior Member, IEEE, \\ Mercè Vall-llossera, Member, IEEE, and Nicolas Reul
}

\begin{abstract}
Aiming to provide sea surface salinity (SSS) maps with a spatiotemporal averaged accuracy of 0.1 psu (practical salinity units), the Soil Moisture and Ocean Salinity (SMOS) community is increasingly focusing on the determination of a robust inversion scheme to enable SSS retrieval from L-band brightness temperature data. In the framework of the Synergetic Aspects and Auxiliary Data Concepts for Sea Surface Salinity Measurements from Space project, efforts have been oriented toward a quantitative analysis of SSS retrieval using different auxiliary data sets. This paper aims to contribute to the assessment of the SMOS salinity retrieval error budget in view of the upcoming SMOS mission ground segment development. Aiming to do that, different models and auxiliary data to simulate and invert the brightness temperature data have been used. An estimation of the different auxiliary parameters' influence has been performed to quantitatively predict at what extent it is reasonable to expect to retrieve salinity once the brightness temperatures are directly measured by the sensor. Statistical distributions of the spatiotemporal averaged errors are provided.
\end{abstract}

Index Terms-Auxiliary data, microwave radiometry, sea salinity, spatiotemporal averaging.

\section{INTRODUCTION}

A CCURATE knowledge of atmospheric and oceanographic processes is crucial for an adequate understanding of the climate system. Within this framework, data collection of ocean salinity fields would arguably have the single most revolutionary impact on the knowledge of conditions that influence global ocean circulation and thus climate [1]. The European Space Agency (ESA) Soil Moisture and Ocean Salinity (SMOS) mission [2] is based on the Microwave Imaging Radiometer by Aperture Synthesis (MIRAS) instrument concept: an Lband two-dimensional (2-D) synthetic aperture radiometer with multiangular and dual-polarimetric/full-polarimetric (dual-pol/ full-pol) imaging capabilities.

Despite the nearly optimal conditions to retrieve salinity from L-band microwave radiometry [3], the sea surface salinity (SSS) signature on brightness temperature $\left(T_{B}\right)$ is still fairly small. Hence, efforts are required to achieve excellent instrument performance and calibration, as well as an optimum definition of the geophysical parameter retrieval scheme. Indeed, the development of a robust and suitable inversion procedure is a challenging key issue for the SMOS community. In

Manuscript received October 7, 2005; revised April 7, 2006.

R. Sabia, A. Camps, and M. Vall-Ilossera are with the Department of Signal Theory and Communications, Polytechnic University of Catalonia, 08034 Barcelona, Spain (e-mail: roberto.sabia@tsc.upc.edu).

N. Reul is with the Département d'Océanographie Physique et Spatiale, Institut Français de Recherche pour l'Exploitation de la Mer, 29280 Plouzané, France.

Digital Object Identifier 10.1109/TGRS.2006.879108 this framework, the main geophysical parameters affecting the brightness temperature variation, and thus having an impact on the SSS retrieval, are sea surface temperature (SST) and wind speed $\left(U_{10}\right)$, the latter being a primary sea-state descriptor. Uncertainties in such required auxiliary parameters may induce errors themselves in the retrieval procedure, hampering reliable salinity estimations.

Within the ESA's Synergetic Aspects and Auxiliary Data Concepts for Sea Surface Salinity Measurements from Space project [4], the evaluation of SSS retrieval uncertainties associated to the use of several different auxiliary data sets has been performed, either concerning SST or wind speed.

This paper is a contribution toward the challenging computation of the SMOS salinity retrieval error budget, which deals with geophysical model functions and retrieval algorithms issues using different auxiliary data sets. Instrumental and other geophysical noise correction sources (mainly atmospheric effects, Sun glint, and Faraday rotation) have been neglected.

In view of the upcoming SMOS mission ground segment development, this paper is meant to approach a quasi-realistic after-launch salinity retrieval setup. Aiming to do this, different models and auxiliary data to simulate and invert the brightness temperature data were used. The analysis of different auxiliary parameters' influence was performed to quantitatively predict at what extent it is reasonable to expect to retrieve salinity once the brightness temperatures are only directly measured by the MIRAS instrument and no longer generated via simulations. Moreover, an item-by-item error source analysis has been done to split the different contributions to the final SSS accuracy error. Spatiotemporal averaged SSS root mean square (rms) accuracy error and bias in a representative area of the North Atlantic Ocean were provided for the different cases studied.

\section{Methodology}

\section{A. SMOS Characteristics}

SMOS single payload, i.e., MIRAS, is a novel radiometer concept that makes use of 2-D aperture synthesis interferometry at L-band to measure the brightness temperatures at two orthogonal polarizations within a wide field of view (FOV) and without any mechanical antenna movement.

MIRAS embodies 69 small receiving antennas uniformly arranged in a Y-shaped array, each of the arms having 21 antennas plus two redundant ones. The total arm length is about $4.5 \mathrm{~m}$, and the instrument's angular resolution is approximately $2^{\circ}$. The interferometric radiometer synthesizes a full image from the cross correlation of simultaneous measurements of the single antenna elements. A snapshot of brightness temperature map of the FOV is obtained every $1.2 \mathrm{~s}$ (integration time for 


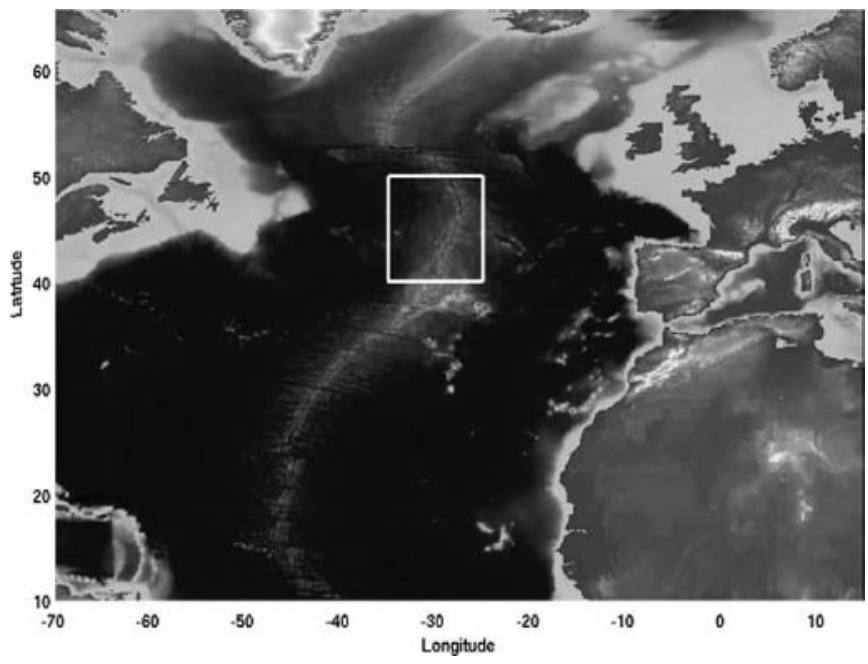

Fig. 1. Mid-Atlantic test zone of $10^{\circ}$ width (longitude $35^{\circ} \mathrm{W}-25^{\circ} \mathrm{W}$, latitude $40^{\circ} \mathrm{N}-50^{\circ} \mathrm{N}$ ).

each polarization) [2]. Due the noncompliance of a Nyquist criterion in the Fourier imaging process, the reconstructed images present aliasing, and the instrument alias-free FOV has a kind of distorted hexagonal shape [5]. In a series of consecutive snapshots, each pixel is observed under different incidence angles, covering at most the range $0^{\circ}-65^{\circ}$, with varying spatial resolution (from 30-60 km). Radiometric accuracy and sensitivity also depend on the position of the pixel in the FOV [6, Fig. 2(b)].

Mission requirements for a $2^{\circ} \times 2^{\circ}$ spatial resolution box after averaging in a ten-day period or for a $1^{\circ} \times 1^{\circ}$ box after averaging in a 30-day period are specified to achieve the Global Ocean Data Assimilation Experiment (GODAE) requirement: a challenging 0.1-psu (practical salinity units) accuracy [17].

\section{B. Auxiliary Data and Generated $T_{B}$ Features}

To evaluate the impact on the retrieved SSS of different auxiliary data, a representative mid-Atlantic test zone (longitude $35^{\circ} \mathrm{W}-25^{\circ} \mathrm{W}$, latitude $40^{\circ} \mathrm{N}-50^{\circ} \mathrm{N}$ ) was chosen (Fig. 1). In this area, there is a small salinity gradient, but a strong wind variability, whose effect on the retrieved SSS was the main issue under study.

Following the recommendations of the other work packages of the project, the selected auxiliary multisource data were the following ${ }^{1}:$ 1) blended QuikSCAT/National Center for Environmental Predictions (NCEP) wind product; 2) European Centre for Medium-Range Weather Forecast (ECMWF) wind product; 3) mean wind field (MWF) QuikSCAT satellite wind product, which were all used as sea surface roughness information (considered as the primary sea-state descriptor); 4) Centre de Météorologie Spatiale (CMS) SST product; 5) NCEP Reynolds SST product; and besides 6) the World Ocean Atlas (WOA) 2001 climatologic SSS field.

${ }^{1}$ Data provided by the Institut Français de Recherche pour l'Exploitation de la Mer (IFREMER).
TABLE I

AUXILIARY Data STATISTICS

\begin{tabular}{|c|c|c|}
\hline Wind Product & Mean Value & Standard Deviation \\
\hline Blended QuikSCAT/NCEP & $11.63 \mathrm{~m} / \mathrm{s}$ & $4.79 \mathrm{~m} / \mathrm{s}$ \\
\hline ECMWF & $10.70 \mathrm{~m} / \mathrm{s}$ & $4.50 \mathrm{~m} / \mathrm{s}$ \\
\hline MWF QuikSCAT & $10.57 \mathrm{~m} / \mathrm{s}$ & $4.01 \mathrm{~m} / \mathrm{s}$ \\
\hline CMS analyzed & $13.50^{\circ} \mathrm{C}$ & $1.68^{\circ} \mathrm{C}$ \\
\hline Reynolds & $13.70^{\circ} \mathrm{C}$ & $1.69^{\circ} \mathrm{C}$ \\
\hline WOA 2001 SSS & $35.68 \mathrm{psu}$ & $0.22 \mathrm{psu}$ \\
\hline
\end{tabular}

The auxiliary blended QuikSCAT/NCEP wind product would be a suitable auxiliary wind parameter for SMOS since it combines numerical weather center products (always available four times a day and close in time to SMOS measurements) with satellite scatterometer data, which inject into the auxiliary product the high wavenumber scales wind energy missing in the numerical product. To analyze the impact of using such consolidated product compared with either numerical weather products alone or scatterometer data alone, the ECMWF product and the daily MWF QuikSCAT product were provided as additional wind auxiliary data.

The use of the analyzed and consolidated GODAE highresolution SST pilot project (GHRSST-PP) type of SST data was recommended for the SMOS Level 2 (L2) processor [4, WP1200]. However, such data are not yet available operationally. Consequently, the analyzed CMS SST product from the Satellite Application Facility/Ocean and Sea Ice (SAF/OSI) was provided since it exhibits strong similarities with the future analyzed GHRSST-PP. An additional and more traditional product, i.e., the weekly NCEP Reynolds SST, was furnished for comparison. Finally, the WOA 2001 monthly climatologic SSS field was used as salinity input in the selected area. Such field exhibits very little variations (standard deviation of the order of $0.2 \mathrm{psu}$ ) with a mean value of $35.7 \mathrm{psu}$ : this shall help in identifying the impact of only auxiliary SST and wind data on the retrieval accuracy. Table I sketches how wind fields present different mean values (their histograms being somewhat shifted among each other) with large standard deviations. Both characteristics will affect the retrieved SSS accuracy, as will be discussed in the following sections [4, WP1400].

Simulated daily brightness temperatures were generated by IFREMER, corresponding to the whole month of January 2003, each time the SMOS instantaneous FOV intersected the region of interest (ROI). Level 1C (geocoded) simulated brightness temperatures were provided for both satellite ascending and descending passes. Time sampling of data along the orbit was set to $1.2 \mathrm{~s}$. Each time an FOV crossed the selected zone, the "Icosahedron Snyder Equal Area hexagonal grid of aperture 4 and resolution 9" (ISEA4H9) grid nodes in the intersection domain were detected, and the auxiliary data closest in time were spatially resampled. From the SMOS observation geometry (incidence and azimuth angles) and the collected auxiliary data sets, the corresponding brightness temperature fields at horizontal and vertical polarizations were computed by means of a direct model. These calculations were performed using the aforementioned blended QuikSCAT wind product, together with CMS SAF/OSI and WOA SSS climatologic field as geophysical inputs within the small-scale approximation [8] direct model for the effect of sea surface roughness on L-band emission. 


\section{Retrieval Algorithm Features}

In this SSS retrieval study, several assumptions have been made concerning the sources of geophysical noise. Faraday rotation was considered to be perfectly known, as well as any atmospheric correction (for a detailed computation of these effects see [9]), being the estimation of such terms out of the scope of this paper, which focuses on the geophysical salinity error budget. Moreover, a perfect sea water dielectric constant model from Klein and Swift [10] was used.

Retrieved SSS variability with respect to different auxiliary parameters was investigated, taking into account the particular SMOS configuration, minimizing the following cost function by means of the Levenberg-Marquardt iterative numerical algorithm [11]:

$$
\begin{aligned}
\varepsilon=\frac{1}{N_{\text {obs }}} \sum_{n}\{ & {\left[\bar{F}_{\text {model }}\left(\theta_{n}, \hat{\vec{P}}\right)-\bar{F}_{\text {data }}\left(\theta_{n}, \vec{P}\right)\right]^{T} } \\
& \times\left(\overline{\bar{C}}_{\text {Full-pol/Dual-pol }}^{\text {Earth } \text { Antenna/odd }(n) \text { even }(n)}\right)^{-1} \\
& \left.\times\left[\bar{F}_{\text {model }}\left(\theta_{n}, \hat{\vec{P}}\right)-\bar{F}_{\text {data }}\left(\theta_{n}, \vec{P}\right)\right]\right\}
\end{aligned}
$$

where $N_{\text {obs }}$ is the number of measurements acquired at the same location in a satellite overpass, $\overline{\bar{C}}$ Earth,Antenna/odd $(n)$, even $(n)$

$\bar{C}_{\text {Full-pol/Dual-pol }}$

is the error covariance matrix that depends on the SMOS operation mode, the reference frame, and the pixel position in the FOV [6]. In turn, $\bar{F}_{\text {model/data }}\left(\theta_{n}, \vec{P}\right)$ is a vector that contains the modeled and measured observables, and its structure depends on the formulation of the retrieval problem. In this paper, the following two formulations are considered:

- $\bar{F}\left(\theta_{n}, \vec{P}\right)=\left[T_{\mathrm{hh}}\left(\theta_{n}, \vec{P}\right), T_{\mathrm{vv}}\left(\theta_{n}, \vec{P}\right)\right]^{T}$, if the problem is formulated in terms of the brightness temperatures in the Earth's reference frame;

- $\bar{F}\left(\theta_{n}, \vec{P}\right)=\left[I\left(\theta_{n}, \vec{P}\right)\right]^{T}=\left[T_{\mathrm{hh}}\left(\theta_{n}, \vec{P}\right)+T_{\mathrm{vv}}\left(\theta_{n}, \vec{P}\right)\right]^{T}=$ $\left[T_{\mathrm{xx}}\left(\theta_{n}, \vec{P}\right)+T_{\mathrm{yy}}\left(\theta_{n}, \vec{P}\right)\right]^{T}$, if the problem is formulated in terms of the first Stokes parameter.

In the first case, to avoid the singularities that appear in the transformation from the antenna to the Earth's reference frame, the MIRAS full-pol mode has been assumed [12]. In the second case, since the first Stokes parameter $I$ can be computed in either the antenna or the Earth's reference frame, the MIRAS dual-pol mode has been assumed since the radiometric noise is lower and to avoid the singularities [6].

In this paper, a restricted version of (1) has been considered, adding further constraints to the minimization algorithm, i.e.,

$$
\begin{aligned}
\varepsilon=\frac{1}{N_{\text {obs }}} \sum_{n}\{[ & \left.\bar{F}_{\text {model }}\left(\theta_{n}, \hat{\vec{P}}\right)-\bar{F}_{\text {data }}\left(\theta_{n}, \vec{P}\right)\right]^{T} \\
& \times\left(\overline{\bar{C}}_{\text {Full-pol/Dual-pol }}^{\text {Earth,Antenna/odd }(n), \text { even }(n)}\right)^{-1} \\
& \left.\times\left[\bar{F}_{\text {model }}\left(\theta_{n}, \hat{\vec{P}}\right)-\bar{F}_{\text {data }}\left(\theta_{n}, \vec{P}\right)\right]\right\} \\
+ & \frac{\left(\mathrm{SSS}-\mathrm{SSS}_{\text {ref }}\right)^{2}}{\sigma_{\text {SSS }}^{2}}+\frac{\left(\mathrm{SST}-\mathrm{SST}_{\text {ref }}\right)^{2}}{\sigma_{\mathrm{SST}}^{2}}+\frac{\left(U_{10}-U_{10_{\text {ref }}}\right)^{2}}{\sigma_{U_{10}}^{2}} .
\end{aligned}
$$

Retrieved SSS estimation is no longer constrained only by searching ranges, but a variable reference value was provided for each parameter, which is weighted by the standard deviation $\left(\sigma_{\text {Aux }}\right)$ of the corresponding auxiliary field (SSS, SST, and $\left.U_{10}\right)$.

The main inversion methodology features are the following.

- Multiparameter retrieval: SSS and both SST and $U_{10}$ were retrieved at once, being adjusted around some reference values to minimize the error.

- Upper and lower boundaries: Physically based "first guess" searching limits are selected, forcing the solution within the chosen ranges. Ranges considered are 25-40 psu for the salinity, $0{ }^{\circ} \mathrm{C}$ to $20{ }^{\circ} \mathrm{C}$ for SST, and 0 to $20 \mathrm{~m} / \mathrm{s}$ for wind speed.

- Auxiliary data standard deviation: A priori knowledge of the calculated standard deviation of each auxiliary data set used in the optimization procedure to suggest the solution once the variability of the auxiliary data is known (Table I). Taking into account that the measurements are neither simultaneous in time nor collocated in space, the chosen metric has been using the spatial variability of the wind field instead of the standard deviation of the error associated with each individual measurement.

- Ten Monte Carlo (MC) simulations of each scenario were performed to estimate another standard deviation, associated with the retrieval at pixel level in the swath, to derive some optimum weights $(1 / \sigma)$ to properly perform the temporal averaging.

- MIRAS operation mode: Full-pol or dual-pol. SSS retrieval can be performed either in full-pol mode using $T_{\mathrm{hh}}$ and $T_{\mathrm{vv}}$ or using the first Stokes parameter $\left(I=T_{\mathrm{hh}}+T_{\mathrm{vv}}=\right.$ $\left.T_{\mathrm{xx}}+T_{\mathrm{yy}}\right)$ in dual-pol mode to optimize the noise.

- Forward/inverse model: Linear fit to the brightness temperature dependence with wind speed from Hollinger [13] is used in the inversion model, on the contrary to the small slope approximation (SSA) method used to generate the brightness temperatures, to stress the fact that the geophysical function will never be perfectly known.

Even if reasonably similar, the brightness temperature sensitivity to wind speed computed with the SSA method and from the linear fit to Hollinger measurements still presents some discrepancies [4, WP1400].

The whole month of simulated $T_{B}$ was analyzed using different combinations of auxiliary data to stress the impact on the retrieved salinity of the different geophysical inputs. The differences with respect to SST or $U_{10}$ are then evaluated.

An assessed retrieval configuration was achieved in two subsequent steps. The first attempt dealt with the restricted version of the cost function described above (2). Nevertheless, simulation results showed that such restrictions in the cost function were too constraining. The retrieved salinity values had a mean value equal to the mean reference value and a standard deviation equal to the variability associated to the input SSS field, thus jeopardizing the attempt to study the SSS sensitivity to the different auxiliary data. Other simulations were performed, either enlarging the climatologic SSS rms value or adding a fake bias, to confirm the previous conclusions.

Analyzing such results, a second satisfactory approach was later identified, in which the SSS was left as a completely free parameter despite of leaving restrictions on SST and $U_{10}$. 

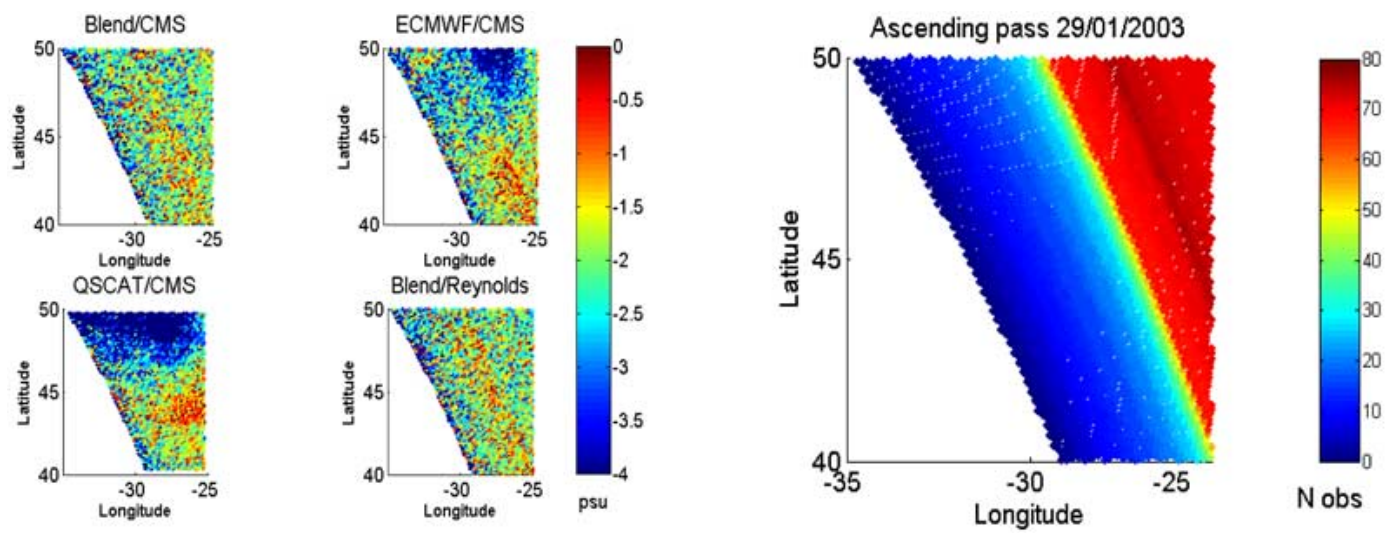

Fig. 2. (a) Salinity single-overpass error maps for different configurations in dual-pol mode using $I$. (b) Number of points used in the retrieval procedure. Both for an ascending pass and corresponding to January 29, 2003.
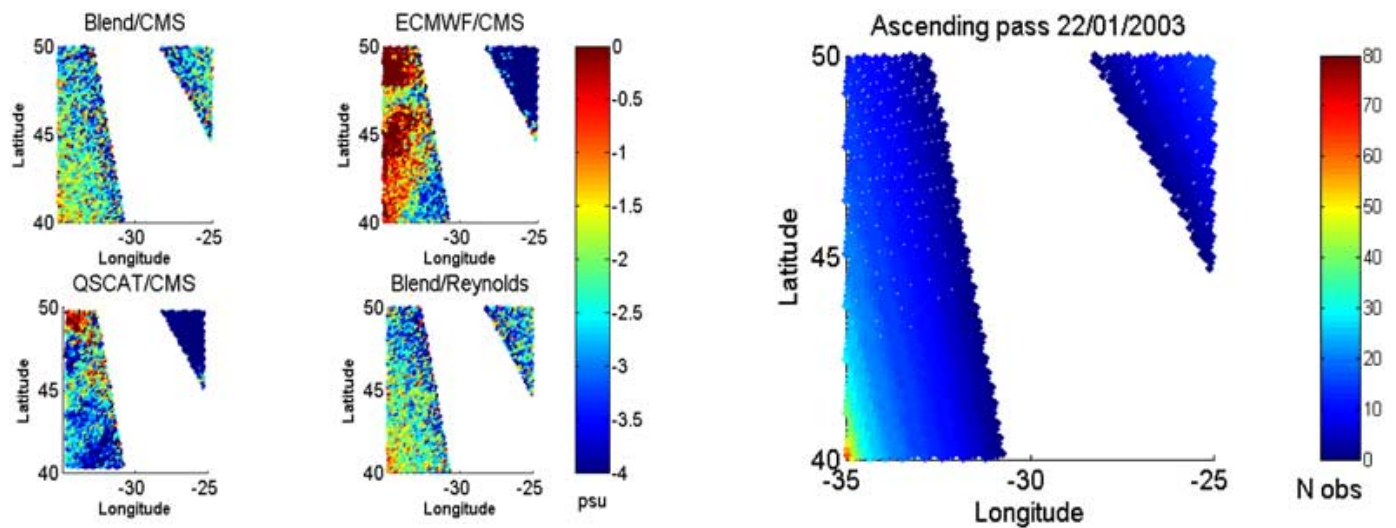

Fig. 3. (a) Salinity single-overpass error maps for different configurations in full-pol mode using $T_{\mathrm{hh}}$ and $T_{\mathrm{vv}}$. (b) Number of points used in the retrieval procedure. Both for an ascending pass and corresponding to January 22, 2003.

Furthermore, simulations have been done as well without any constraint except for the upper and lower boundaries (1) as previously done in [14] and [15].

\section{SALinity Retrieval}

\section{A. Single Overpass}

As mentioned before, the first version of the algorithm (with restrictions on SSS, SST, and $U_{10}$ ) hampered the analysis of the retrieved variation due to different auxiliary data. In the second approach, simulations have been performed for four different auxiliary data couples, the two instrument operation modes (full-pol and dual-pol) and separated satellite passes (ascending and descending), resulting in 16 monthly data sets to be processed. The track separation was needed: 1) since SST gradients may be different at 6 A.M. and at 6 P.M. and 2) to avoid averaging pixels imaged in a different position within the swath in the two satellite passes. Yet, other simulations were carried out without considering any reference value, i.e., no auxiliary parameters were introduced in the minimization procedure, which converged without any constraints.

Four different configurations were identified, which aim at stressing the variability, first, with respect to auxiliary wind (blended wind, ECMWF, and QuikSCAT), keeping constant SST, and, second, with respect to a different SST field (Reynolds), assuming default blended wind.
Fig. 2(a) shows error maps (difference between the original and retrieved salinities) corresponding to different configurations using the first Stokes parameter measured in dual-pol mode for an ascending pass corresponding to January 29. The visibility of satellite's swath is clearly seen, as well as the SSS error variability with respect to the different auxiliary data. Fig. 2(b) plots the corresponding number of times each pixel is imaged [ $N_{\text {obs }}$ in (1) and (2)].

Fig. 3(a) and (b), in turn, is related to a different day (January 22) and to full-pol mode. Again, SSS errors at pixel level are the representative values of single-overpass salinity retrieval. This example was selected to underline how in some days the satellite covered only partially the ROI, with pixels being imaged only very few times. This implied very noisy measurements, leading to the need of a weighted mean to properly perform the temporal averaging.

\section{B. Temporal Averaging}

To develop preliminary concepts for processing the auxiliary data in preparation of SMOS L2 ground segment implementation, spatiotemporal averaging has been approached as follows. Concerning the temporal averaging, the aforementioned SSS errors (single overpass) have been averaged at the pixel level along the whole month. However, provided the huge day-to-day variability of these errors due to the different pixel positions 


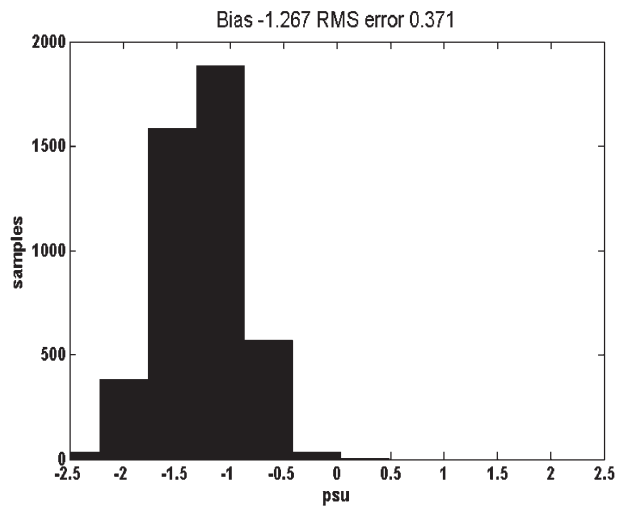

(a)

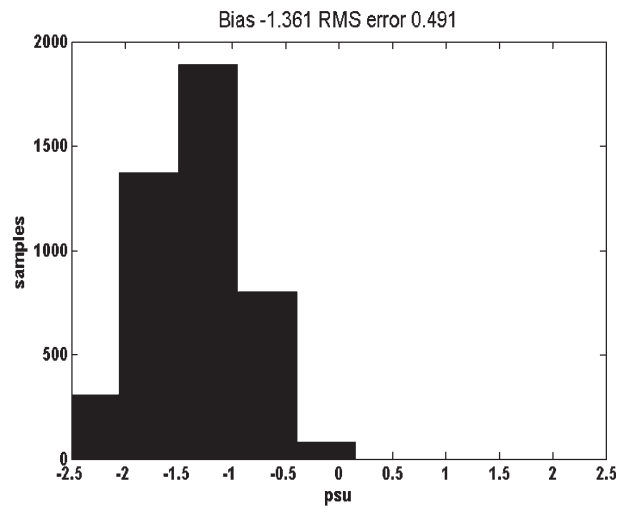

(b)

Fig. 4. (a) Monthly SSS weighted error histogram with ROI bias and rms accuracy referring to blend/CMS configuration in dual-pol mode. (b) Monthly SSS weighted error histogram with ROI bias and rms accuracy referring to ECMWF/CMS configuration in dual-pol mode.

within the FOV (distance to the satellite's ground track), a weighted mean was needed. Such weights were computed as the inverse of the standard deviations calculated from the different realizations of each pixel (ten MC realizations). Thus, each single-pixel retrieval value was used to obtain the weights for an adequate evaluation of the monthly error at pixel level. These weights are dependent on the cross-track distance and could be used in the operational processor. Pixels with retrieved SSS error farther away than \pm 2.5 psu from the most probable value were discarded as wrong and not averaged. Afterward, an overall mean and rms at ROI level allowed computing the expected bias and rms accuracy of each time-analyzed configuration.

Fig. 4(a) shows the histogram of the weighted errors within the ROI for the blend/CMS (cf. auxiliary data listed in the previous section) configuration in the ascending passes. The monthly expected bias and the rms accuracy were underlined, the latter taken as retrieval goodness index in this paper. This bias, being quite homogeneous in the whole ROI, could be potentially compensated for by means of an external calibration using moored buoys or drifters as proposed in [15].

In turn, Fig. 4(b) refers to the same procedure, but using a different wind auxiliary data set from ECMWF. As it can be seen, this configuration exhibits quite different values for the expected bias and the rms accuracy from the previous one; both the bias and the standard deviation are worse than in the previous configuration.

It has to be stressed, however, that the blend/CMS configuration represents the ideal case, being the default auxiliary data used in the brightness temperatures generation with the SSA direct model, and all the retrievals obtained using other data combinations are supposed to be worse. On the other hand, one of the goals of the study was to quantitatively check how good this retrieval could be considering that different models to generate and invert the data (more realistic case) are used. Furthermore, taking the default configuration as a baseline, the study focused on the evaluation of the worsening of retrieval accuracy once different auxiliary data set are considered. Addressing SSS retrieval using other than default auxiliary data is an attempt to quantify the error accuracy to be realistically expected, at least concerning scene-related issues (apart from other geophysical noise contributions).

\section{Spatial Averaging}

Once the temporal averaging has been studied for different configurations, a spatial averaging is conducted in $1^{\circ} \times 1^{\circ}$ and $2^{\circ} \times 2^{\circ}$ boxes, being identified as acceptable spatial resolution [7]. Single-pixel monthly weighted errors coming from temporal processing have been sorted according to their geographic locations within the ROI and then averaged in single boxes.

Table II summarizes the results gathered so far for each configuration. Several considerations arise from the results shown in the table. As expected, concerning the auxiliary wind impact, ECMWF and QuikSCAT winds turned out to be worse than blended wind product (default wind used in brightness temperature generation). Nevertheless, quantitative degradation (i.e., SSS sensitivity to auxiliary wind) with respect to the baseline configuration has been provided.

Concerning the impact of the auxiliary SST, a minimum effect is encountered comparing the results using the Reynolds field and the default CMS field. One possible reason is that the rms values considered in the algorithm restrictions for both fields are really close. Furthermore, $T_{B}$ exhibits a low sensitivity with respect to SST around 35 psu [14].

The use of $T_{\mathrm{hh}}$ and $T_{\mathrm{vv}}$ measured in full-pol mode provides a slightly better rms accuracy but a slightly worse bias than using the first Stokes parameter measured as $I=T_{\mathrm{xx}}+T_{\mathrm{yy}}$ in dualpol mode.

Concerning the unrestricted retrieval algorithm version (no reference values), the use of $I$ measured in dual-pol mode provides a lower bias even if, as expected, the rms error is larger. Regarding the last consideration, a possible suggestion to correct for the bias encountered in the restricted configurations might be to perform the retrieval first with an unrestricted configuration and with the first Stokes parameter (lower bias), then moving to the restricted (with respect to SST and $U_{10}$ ) version to obtain better SSS retrieval accuracy performances, and then matching the mean values.

Concerning spatiotemporal SSS rms accuracy, as it can be appreciated, the bias is obviously the same, but the rms accuracy has not decreased as expected, taking into account the number of available observations. The expected reduction is not seen since the retrieval often presented errors in the form of patches, which reduced the efficiency of the averaging procedure. 
TABLE II

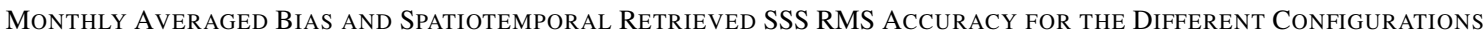
(IN BOLD: SMALleSt SSS ERROR; IN ITALIC: CONFIGURATIONS SATISFYING GODAE REQUiREMENTS)

\begin{tabular}{|c|c|c|c|c|c|c|}
\hline Configuration & Pol. Mode & $\begin{array}{c}\text { Satellite } \\
\text { pass }\end{array}$ & $\begin{array}{c}\text { Monthly } \\
\text { Bias }\end{array}$ & $\begin{array}{c}\text { Monthly } \\
\text { Rms } \\
\text { accuracy }\end{array}$ & $\operatorname{Rms} 1^{o} x 1^{o}$ & $\operatorname{Rms} 2^{\circ} \times 2^{\circ}$ \\
\hline Blend/CMS & Dual (Stokes I) & UP & -1.267 & 0.371 & 0.071 & 0.050 \\
\hline Blend/CMS & Dual (Stokes I) & DN & -1.311 & 0.382 & 0.099 & 0.080 \\
\hline ECMWF/CMS & Dual (Stokes I) & UP & -1.361 & 0.491 & 0.264 & 0.248 \\
\hline ECMWF/CMS & Dual (Stokes I) & $\mathrm{DN}$ & -1.593 & 0.549 & 0.293 & 0.273 \\
\hline QSCAT/CMS & Dual (Stokes I) & UP & -1.472 & 0.546 & 0.309 & 0.291 \\
\hline QSCAT/CMS & Dual (Stokes I) & $\mathrm{DN}$ & -1.530 & 0.513 & 0.206 & 0.162 \\
\hline Blend/Reynolds & Dual (Stokes I) & UP & -1.258 & 0.368 & 0.078 & 0.057 \\
\hline Blend/Reynolds & Dual (Stokes I) & $\mathrm{DN}$ & -1.316 & 0.395 & 0.102 & 0.081 \\
\hline No aux data & Dual (Stokes I) & UP & -0.860 & 1.505 & 0.835 & 0.737 \\
\hline No aux data & Dual (Stokes I) & DN & -0.791 & 1.195 & 0.431 & 0.326 \\
\hline Blend/CMS & Full (Th/Tv) & UP & -1.447 & 0.337 & 0.055 & 0.036 \\
\hline Blend/CMS & Full (Th/Tv) & DN & -1.462 & 0.331 & 0.061 & 0.032 \\
\hline ECMWF/CMS & Full (Th/Tv) & UP & -1.512 & 0.401 & 0.186 & 0.166 \\
\hline ECMWF/CMS & Full (Th/Tv) & $\mathrm{DN}$ & -1.679 & 0.443 & 0.212 & 0.198 \\
\hline QSCAT/CMS & Full (Th/Tv) & UP & -1.584 & 0.469 & 0.234 & 0.220 \\
\hline QSCAT/CMS & Full (Th/Tv) & $\mathrm{DN}$ & -1.622 & 0.411 & 0.136 & 0.097 \\
\hline Blend/Reynolds & Full (Th/Tv) & UP & -1.443 & 0.346 & 0.070 & 0.045 \\
\hline Blend/Reynolds & Full (Th/Tv) & $\mathrm{DN}$ & -1.453 & 0.330 & 0.056 & 0.035 \\
\hline No aux data & Full (Th/Tv) & UP & -3.457 & 0.902 & 0.289 & 0.207 \\
\hline No aux data & Full (Th/Tv) & $\mathrm{DN}$ & -3.295 & 0.893 & 0.259 & 0.211 \\
\hline
\end{tabular}

TABLE III

Progressive ERror SOURCE IDENTIFICATION SCHEME

\begin{tabular}{|l|c|c|c|c|c|}
\hline Types & $\begin{array}{c}\text { Radiometric } \\
\text { Noise }\end{array}$ & $\begin{array}{c}\text { Model } \\
\text { Difference }\end{array}$ & $\begin{array}{c}\text { Ref. Point } \\
\text { Uncertainty }\end{array}$ & $\begin{array}{c}\text { Auxiliary Data } \\
\text { Fields }\end{array}$ & Features \\
\hline Type 1 & No & No & No & $35 \mathrm{psu}, 15^{\circ} \mathrm{C}, 10 \mathrm{~m} / \mathrm{s}$ & Ideal \\
\hline Type 2 & Yes & No & No & $35 \mathrm{psu}, 15^{\circ} \mathrm{C}, 10 \mathrm{~m} / \mathrm{s}$ & Noise \\
\hline Type 3 & No & No & Yes & $35 \mathrm{psu}, 15^{\circ} \mathrm{C}, 10 \mathrm{~m} / \mathrm{s}$ & Ref point effect \\
\hline Type 4 & No & Yes & Yes & Same (Default) & Model diff effect \\
\hline Type 5 & Yes & Yes & Yes & Same (Default) & Blended/CMS \\
\hline Type 6 & Yes & Yes & Yes & Different & Different Aux Data \\
\hline
\end{tabular}

\section{IDENTIFICATION OF RETRIEVAL ERROR SOURCES}

Having the overall spatiotemporal retrieved SSS variability scheme at our disposal, it is interesting to quantitatively and singularly identify the different sources of SSS bias and rms accuracy error. The aim is to distinguish between the variability associated with different auxiliary data (main issue addressed in this paper) from the additional different contributions involved in the inversion procedure itself.

A master retrieval scenario referring to the ascending passes in dual-pol mode and using the first Stokes parameter was selected, where the possible additional error sources where binned into radiometric noise, direct/inverse model difference, uncertainties on auxiliary data, and auxiliary wind/SST difference.

As a first approach, auxiliary data difference was not considered since salinity, temperature, and wind fields were considered constant in the whole ROI $\left(35 \mathrm{psu}, 15^{\circ} \mathrm{C}\right.$, and $\left.10 \mathrm{~m} / \mathrm{s}\right)$, both to generate brightness temperatures (via the Hollinger model) and to restrict the cost function.

Table III summarizes the simulation plan conceived with the purpose of generating a sequence of simulations with an increasing complexity to furnish a tool to quantify the multisource error contributions in the results obtained so far.

The simplest simulation case to test the reliability of the iterative method did not embody any of the aforementioned potential error sources: there was no radiometric noise, and a
Hollinger linear model was used both for generating $T_{B}$ and for inverting data (direct and inverse model), while reference points in the cost function restrictions (actually a varying value with the corresponding uncertainties) were supposed to be known exactly (type 1). As expected, in such ideal conditions, SSS retrieval was perfect, only limited by numerical roundoff errors, confirming the robustness of the method.

The second step, labeled as type 2, consisted of adding only the expected radiometric noise. At boresight, the considered noise was $2.36 \mathrm{~K}$, with a degradation factor toward the swath edges, which is a function of the so-called "obliquity factor" and the antenna radiation patterns. The corresponding results exhibit good retrieval, with the expected worsening toward the edges of the swath due to the larger noise and reduced number of observations.

Subsequent simulations were devoted to the understanding of the magnitude of the auxiliary data uncertainties (type 3). Again, radiometric noise was absent, and direct/inverse models were the same; but the restrictions in the reference points in the cost function are now supposed to be known with some degree of uncertainty, namely the a priori considered rms of the default wind and SST fields. The temporal averaged results shown hereafter emphasize how this configuration is not responsible for bias introduction in SSS retrieval, while it already contributes with a monthly rms SSS error of 0.293 psu [Fig. 5(a)]. 


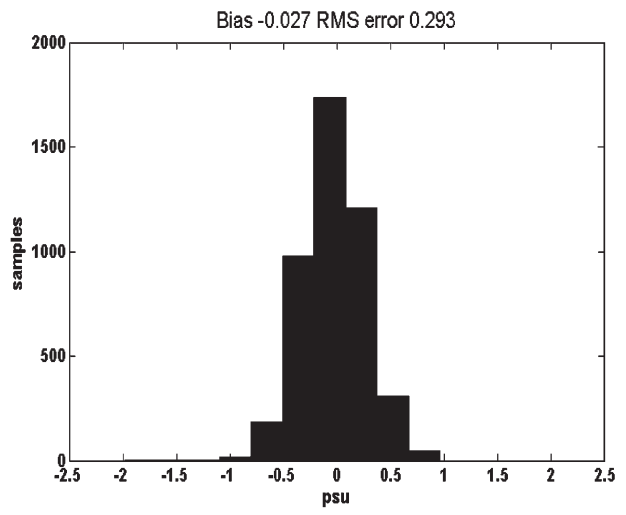

(a)

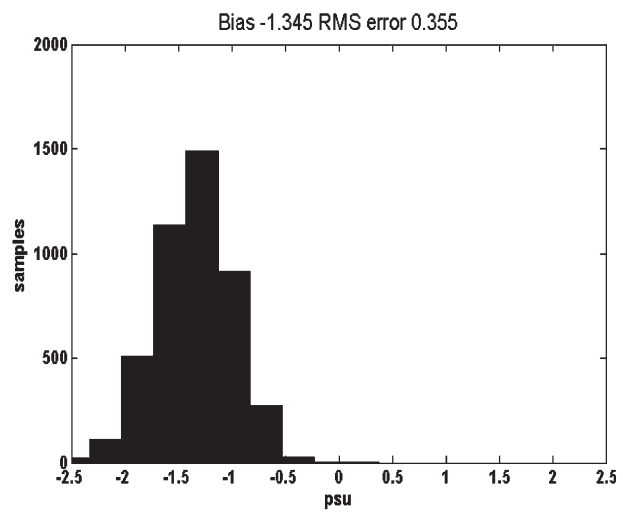

(b)

Fig. 5. (a) Monthly SSS weighted error histogram with ROI bias and rms accuracy referring to type 3 configuration in dual-pol mode. (b) Monthly SSS weighted error histogram with ROI bias and rms accuracy referring to type 4 configuration in dual-pol mode.

TABLE IV

QUANTITATIVE ERROR CONTRIBUTION

\begin{tabular}{|c|c|c|c|c|c|c|}
\hline Configuration & Pd. Mode & $\begin{array}{c}\text { Satellite } \\
\text { pass }\end{array}$ & $\begin{array}{c}\text { Monthly } \\
\text { Bias }\end{array}$ & $\begin{array}{c}\text { Monthly } \\
\text { Rms } \\
\text { accuracy }\end{array}$ & $\operatorname{Rms} 1^{\circ} x 1^{o}$ & $\operatorname{Rms} 2^{\circ} \times 2^{\circ}$ \\
\hline Type 1 & (I) & UP & 0.000 & 0.000 & 0.000 & 0.000 \\
\hline Type 2 & (I) & UP & 0.008 & 0.130 & 0.033 & 0.024 \\
\hline Type 3 & (I) & UP & -0.027 & 0.293 & 0.046 & 0.025 \\
\hline Type 4 & (I) & UP & -1.345 & 0.355 & 0.083 & 0.064 \\
\hline $\begin{array}{l}\text { Type } 5 \\
\text { Blended }\end{array}$ & (I) & UP & -1.267 & 0.371 & 0.071 & 0.050 \\
\hline $\begin{array}{c}\text { Type } 6 \\
\text { ECMWF }\end{array}$ & (I) & UP & -1.361 & 0.491 & 0.264 & 0.248 \\
\hline $\begin{array}{c}\text { Type } 6 \\
\text { QuikSCAT }\end{array}$ & (I) & UP & -1.472 & 0.546 & 0.309 & 0.291 \\
\hline
\end{tabular}

Keeping in mind the increasing degree of complexity to gradually approach the overall setup used in this paper, the difference between direct and inverse models was then added to the previous configuration (type 4). In this case, auxiliary data are no longer the constant fields used until now in this section, but the default blended wind product and CMS temperature. Fig. 5(b) illustrates the monthly averaged results for this case.

Table IV compares the spatiotemporal averaged results through type 1 to type 6 cases. It is useful to recall that the first one is the ideal case, while the second one evaluates the presence of radiometric noise. Type 3 underlines the reference point uncertainty effect; type 4 emphasizes the model difference impact, and finally; type 5 is the already known blend/CMS default configuration (cf. Section III). Two examples of type 6 different auxiliary data configuration are listed as well.

Comparing type 4 and type 5 configurations, the bias appears slightly higher $(-1.345 \mathrm{psu})$ in the first one, whereas the rms accuracy is slightly better $(0.355 \mathrm{psu})$ in time-averaged results but moderately worse in the space-averaged retrievals, indicating that, as compared with other error sources, the radiometric noise does not affect results heavily. The type 6 case, as reported in Table IV, refers to the auxiliary data difference effect, whose magnitude on SSS was already the matter of study throughout this paper. As mentioned in the previous sections, if one refers to type 6 data as a "realistic" retrieval case and assuming that the different contributions are independent, it is now possible to unwrap the overall SSS error coming from different sources. For instance, considering ECMWF monthly accuracy and $1^{\circ} \times 1^{\circ}$ spatial averaging, $\sim 2 \%$ of the final squared error lies in the ra- diometric noise, an additional $\sim 3 \%$ is related to reference point variability, an extra $\sim 7 \%$ is due to the model difference, while the remaining $\sim 88 \%$ is associated to auxiliary data uncertainty. In the case of QuikSCAT, the degradation effect due to auxiliary data difference is $\sim 92 \%$ of the total squared error.

It has to be stressed, however, that part of the SSS additional error coming from the last step might be related to unacceptable original/auxiliary wind difference as well. Fig. 6(a) depicts an example of absolute difference between wind used in the $T_{B}$ generation (default blended QSCAT/NCEP) and auxiliary wind used in the minimization procedure (in this case, ECMWF). Such difference can be very high and induce strong discrepancies between the original and the retrieved SSS, according to geographical zone.

The corresponding SSS errors are shown in Fig. 6(b), which referred to ascending pass in dual-pol mode. Note that SSS exhibits remarkable errors in correspondence of the ROI zone characterized for high original/auxiliary wind difference.

In summary, the influence of each parameter has been studied starting from an ideal simulation, adding then separately radiometric noise and uncertainties on auxiliary data, respectively. Hence, considering the latter one as always present, the effect of having different models has been investigated. Finally, the progressive impact to SSS bias and rms error has been quantified, performing a proper identification of the different error contributions.

Once it is clear that bias encountered in the retrieved salinity lies mostly in the use of a different model for inverting data, a further attempt was made substituting the Hollinger linear fit used in the study with a different empirical model, namely 

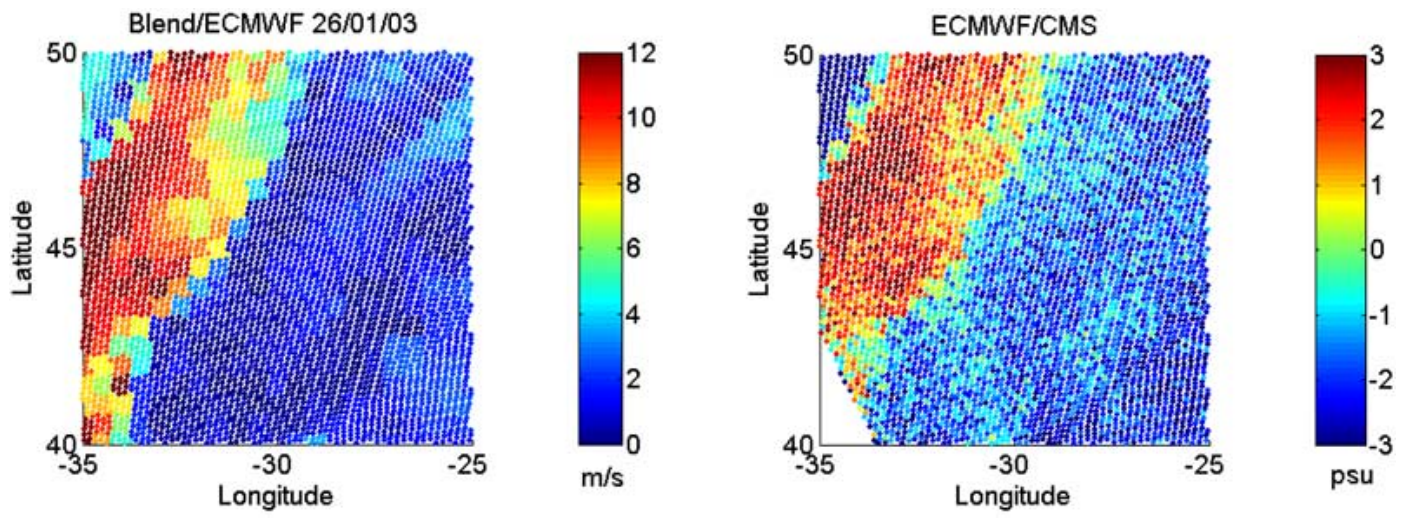

Fig. 6. (a) Absolute difference (in meters per second) between blended QSCAT/NCEP and ECMWF auxiliary winds for January 26. (b) Corresponding retrieved SSS errors for January 26, ascending pass, dual-pol, and ECMWF auxiliary data.

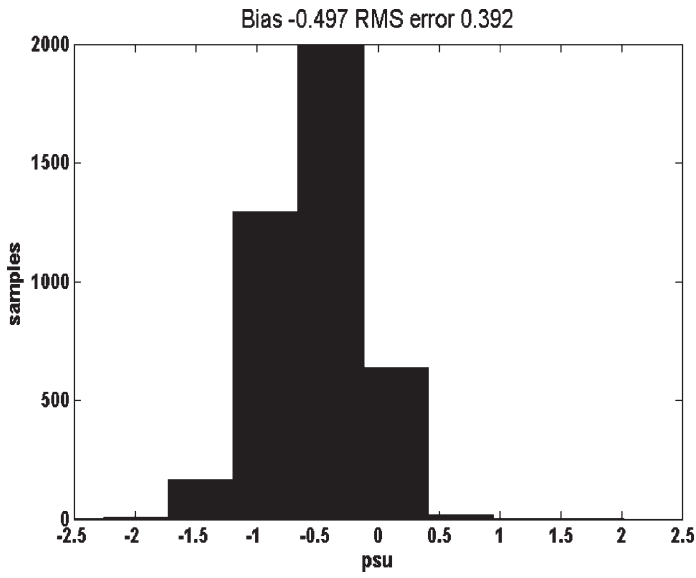

Fig. 7. Monthly SSS weighted error histogram with ROI bias and rms accuracy using WISE model in dual-pol mode.

the WISE 2001 [16] one. Results are shown in Fig. 7. Bias has been drastically reduced (from -1.267 psu using the linear fit to Hollinger model to -0.497 psu using the WISE 2001 model), indicating that obtaining a good forward model for the roughness effect on sea surface emissivity is a key issue that deserves the maximum attention and effort.

\section{Conclusion}

The aim of this paper was twofold.

1) To analyze the impact of the use of different auxiliary data sets in the retrieval process, quantifying the sensitivity to the auxiliary fields, and to check the quality of the achievable results using different brightness temperature models to generate/invert the data.

2) To identify the different SSS accuracy contributions through an item-by-item error source selection that is meant to distinguish between the auxiliary data impact and the additional contributions involved in the inversion procedure itself.

The use of $T_{\mathrm{hh}}$ and $T_{\mathrm{vv}}$ measured in full-pol mode has been shown to provide a slightly better rms SSS error by a varying factor, mostly ranging from 1.1 to 1.2 , but a slightly worse bias than using the first Stokes parameter measured as $I=T_{\mathrm{xx}}+$ $T_{\mathrm{yy}}$ in dual-pol mode.
The main conclusions can be summarized as follows.

- SSS retrieval in one overpass: RMS SSS error is typically around 1 psu (varying depending on the auxiliary data used), increasing up to 4 psu or more at swath edges, which is in agreement with [14], [17], and [18]. A bias appears in the measurements and must be corrected for by using ground-truth data (e.g., buoys or drifters).

- Temporal averaged retrieved SSS: Monthly temporal averaging at pixel level provides an ROI rms error within the range $0.330-0.549 \mathrm{psu}$, depending on the auxiliary data set used. The use of different sources of auxiliary data for SST has a minimum impact in the SSS retrieval.

- Spatiotemporal averaging of retrieved SSS: In a period of 30 days and in $1^{\circ} \times 1^{\circ}$ boxes, the retrieved rms SSS error ranges between 0.055 and 0.309 psu. In a period of 30 days and in $2^{\circ} \times 2^{\circ}$ boxes, the retrieved rms SSS accuracy ranges between 0.032 and 0.291 psu. The best SSS products obtained by spatiotemporal averaging of 30 days satisfy the $<0.1$-psu error requirement.

Apart from the best case scenario that determines the limits of the retrieval, if ECMWF data were used instead (different auxiliary wind), the rms error would increase up to $0.2-0.3 \mathrm{psu}$ (depending on instrument's configuration and satellite pass), which is in accordance with other studies [15]. These studies have shown that without auxiliary data and after spatiotemporal averaging ( 30 days, $1^{\circ} \times 1^{\circ}$ ), the SSS rms error ranges from $0.2 \mathrm{psu}$ at the equator to $0.7 \mathrm{psu}$ in polar regions. To address an even more realistic case (besides model difference), one should refer to different auxiliary data configurations since it better simulates the upcoming "measured" brightness temperature.

Identification of retrieval error sources has also been performed by isolating potential discrepancy sources. Reference point variability, which is linked to the auxiliary field uncertainty, determines a significant part of the final error even if it does not produce bias. Direct/inverse model difference, in turn, seems to be responsible for bias introduction besides some extra contribution to the SSS error. In this context, radiometric noise does not seem to play a crucial role since, apparently, its effect is mostly filtered out in the spatiotemporal averaging procedure. Apart from the intrinsic auxiliary wind/SST difference, additional residual error sources lie in biased auxiliary MWF values (Table I) and in zonal strong inhomogeneities between winds [Fig. 6(a)]. 
Concerning the bias introduced by model differences, an attempt of replacing the Hollinger model with the WISE 2001 model provided significant bias reduction, stressing how obtaining a good forward model for the roughness effect on sea surface emissivity is still a key issue in the retrieval procedure.

\section{ACKNOWLEDGMENT}

The authors would like to thank three anonymous reviewers for their comments and suggestions that helped improve the clarity of this paper. This paper has been mainly developed within the ESA's Synergetic Aspects and Auxiliary Data Concepts for Sea Surface Salinity Measurements from Space project [4], whose participants are thanked for their useful comments during the project meetings.

\section{REFERENCES}

[1] P. Silvestrin, M. Berger, Y. Kerr, and J. Font, "ESA's second Earth Explorer Opportunity mission: The Soil moisture and Ocean Salinity mission-SMOS," IEEE Geosci. Remote Sens. Newslett., no. 118, pp. 11-14, Mar. 2001

[2] J. Font, G. Lagerloef, D. LeVine, A. Camps, and O. Z. Zanife, "The determination of surface salinity with the European SMOS space mission," IEEE Trans. Geosci. Remote Sens., vol. 42, no. 10, pp. 2196-2205, Oct. 2004.

[3] C. T. Swift and R. E. McIntosh, "Considerations for microwave remote sensing of ocean-surface salinity," IEEE Trans. Geosci. Remote Sens., vol. GRE-21, no. 4, pp. 480-491, Jul. 1983.

[4] "Synergetic aspects and auxiliary data concepts for sea surface salinity measurements from space," ESA, Noordwijk, The Netherlands, ESA ESTEC 18176/04/NL/CB, Jan. 2006. Final Report. [Online]. Available:

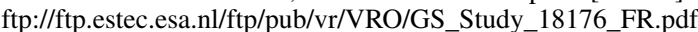

[5] A. Camps, J. Bará, I. Corbella, and F. Torres, "The processing of hexagonally sampled signals with standard rectangular techniques: Application to 2-D large aperture synthesis interferometric radiometers," IEEE Trans. Geosci. Remote Sens., vol. 35, no. 1, pp. 183-190, Jan. 1997.

[6] A. Camps, M. Vall-llossera, N. Duffo, F. Torres, and I. Corbella, "Performance of sea surface salinity and soil moisture retrieval algorithms with different ancillary data sets in 2-D L-band aperture synthesis interferometric radiometers," IEEE Trans. Geosci. Remote Sens., vol. 43, no. 5, pp. 1189-1200, May 2005.

[7] N. Smith and M. Lefèbvre, "The Global Ocean Data Assimilation Experiment (GODAE); Monitoring the oceans in the 2000s: An integrated approach," in Proc. Int. Symp., Biarritz, France, Oct. 15-17, 1997.

[8] N. Reul and B. Chapron, "SMOS salinity data processing study: Improvements in emissivity models," Final Rep. WP 1100. ESA, Nordwijk, The Netherlands. Contract 15165/01/NL/SF.

[9] N. Skou and D. Hoffman-Bang, "L-band radiometers measuring salinity from space: Atmospheric propagation effects," IEEE Trans. Geosci. Remote Sens., vol. 43, no. 10, pp. 2210-2217, Oct. 2005.

[10] L. A. Klein and C. T. Swift, "An improved model for the dielectric constant of sea water at microwave frequencies," IEEE J. Ocean. Eng., vol. OE-2, no. 1, pp. 104-111, 1977.

[11] W. Press, S. Teukolsky, W. Vetterling, and B. Flannery, Numerical Recipes in C: The Art of Scientific Computing, 2nd ed. New York: Cambridge Univ. Press, 1992.

[12] M. Martin-Neira, S. Ribó, and A. J. Martin-Polegre, "Polarimetric mode of MIRAS," IEEE Trans. Geosci. Remote Sens., vol. 40, no. 8, pp. 1755-1768, Aug. 2002.

[13] J. P. Hollinger, "Passive microwave measurements of sea surface roughness," IEEE Trans. Geosci. Electron., vol. GE-9, no. 3, pp. 165-169, 1971.

[14] A. Camps, I. Corbella, M. Vall-llossera, N. Duffo, F. Torres, R. Villarino, L. Enrique, F. Julbé, J. Font, A. Julià, C. Gabarró, J. Etcheto, J. Boutin, A. Weill, V. Caselles, E. Rubio, P. Wursteisen, and M. Martín-Neira, "L-band sea surface emissivity: Preliminary results of the WISE-2000 campaign and its application to salinity retrieval in the SMOS mission," Radio Sci., vol. 38, no. 4, p. 8071, Jun. 2003.

[15] A. Camps, M. Vall-llossera, N. Duffo, F. Torres, and I. Corbella, "Retrieving sea surface salinity with multi-angular L-band brightness temperatures: Improvement by spatio-temporal averaging," Radio Sci., vol. 40, no. 2, RS2003, 2005.

[16] A. Camps, J. Font, M. Vall-1lossera, C. Gabarró, I. Corbella, N. Duffo, F. Torres, S. Blanch, A. Aguasca, R. Villarino, L. Enrique, J. Miranda, J. Arenas, A. Julià, J. Etcheto, V. Caselles, A. Weill, J. Boutin,
S. Contardo, R. Niclós, R. Rivas, S. C. Reising, P. Wursteisen, M. Berger, and M. Martín-Neira, "The WISE 2000 and 2001 campaigns in support of the SMOS mission: Sea surface L-band brightness temperature observations and their application to multi-angular salinity retrieval," IEEE Trans. Geosci. Remote Sens., vol. 42, no. 4, pp. 804-821, Apr. 2004.

[17] J. Boutin et al., "Surface salinity retrieved from SMOS measurements over the global ocean: Imprecisions due to sea surface roughness and temperature uncertainties," J. Atmos. Ocean. Technol., vol. 21, no. 1, pp. 1432-1447, 2004.

[18] A. Camps, N. Duffo, M. Vall-llossera, and B. Vallespín, "Sea surface salinity retrieval using multi-angular L-band radiometry: Numerical study using the SMOS end-to-end performance simulator," in Proc. IGARSS, Toronto, ON, Canada, 2002, vol. 2, pp. 1123-1125.

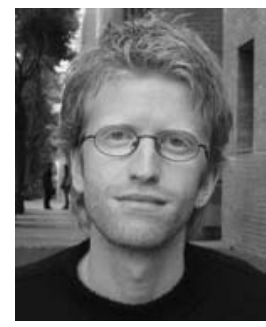

Roberto Sabia (S'03) was born in Naples, Italy, on November 13, 1975. He received the degree in marine environmental sciences (cum laude), curriculum in oceanography (five-year course study), from the Università degli Studi di Napoli "Parthenope," Naples, in 2002. He is currently working toward the Ph.D. degree in signal theory and communications at the Polytechnic University of Catalonia (UPC), Barcelona, Spain.

In 2002, he was a recipient of a Grant on sea surface scattering models at the Microwave Remote Sensing Laboratory, Università degli Studi di Napoli "Parthenope." In 2003 , he joined the Department of Signal Theory and Communications, UPC. He participated in the FROG 2003, REFLEX 2003, and MOUSE 2004 field campaigns and participated in two ESA studies within the frame of the SMOS Earth Explorer Mission. His main research interests deal with microwave radiometry and sea surface salinity retrieval.

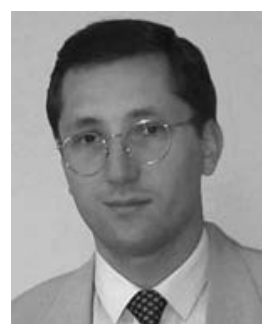

Adriano Camps (S'91-A'97-M'00-SM'03) was born in Barcelona, Spain, in 1969. He received the degree in telecommunications engineering and the $\mathrm{Ph} . \mathrm{D}$. degree in telecommunications engineering both from the Polytechnic University of Catalonia (UPC), Barcelona, in 1992 and 1996, respectively.

From 1991 to 1992, he was with the ENS des Télécommunications de Bretagne, Bretagne, France, with an Erasmus Fellowship. In 1993, he joined the Electromagnetics and Photonics Engineering group, at the Department of Signal Theory and Communications, UPC, as an Assistant Professor, and since 1997, as an Associate Professor. In 1999, he was on sabbatical leave at the Microwave Remote Sensing Laboratory, University of Massachusetts, Amherst. His research interests are microwave remote sensing, with special emphasis on microwave radiometry by aperture synthesis techniques. He has performed numerous studies within the frame of the European Space Agency's SMOS Earth Explorer Mission. He is an Associate Editor of Radio Science.

Dr. Camps received the second national award of university studies in 1993; the INDRA award of the Spanish Association of Telecommunication Engineering to the best Ph.D. in 1997; the extraordinary Ph.D. award at the Universitat Politècnica de Catalunya in 1999; the First Duran Farell Award and the Ciudad de Barcelona Award, in 2000 and 2001, respectively, both for Technology Transfer; and the Research Distinction of the Generalitat de Catalunya for contributions to microwave passive remote sensing in 2002 . $\mathrm{He}$ also won the "Premi Nacional de Telecomunicacions 2003" and "Premi Salvà i Campillo 2004 and the European Young Investigator (EURYI) Award in 2004." $\mathrm{He}$ was the Chair of $\mu \mathrm{Cal}$ ' 01 . He is the Editor of the IEEE GEOSCIENCE ANd Remote Sensing Newsletter and President-Founder of the IEEE Geoscience And Remote Sensing Society Chapter in Spain. 


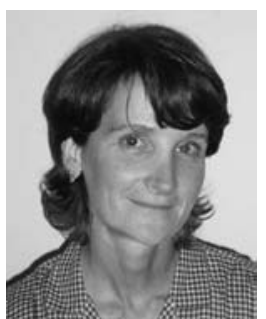

Mercè Vall-llossera (M'99) received the senior telecommunication engineering and the Doctor telecommunication engineering degrees from the Polytechnic University of Catalonia (UPC), Barcelona, Spain, in 1990 and 1994, respectively.

She has been lecturing and doing research at the Department of Signal Theory and Communications, UPC, from 1990 to 1997, as an Assistant Professor, and since 1997, as an Associate Professor. She spent a sabbatical year in Montreal with the scholarship of the "Programme Québécois de Bourses d'Excellence" (1996-1997): "Stages de Formation postdoctorale au Québec pour jeunes diplômés étrangers." Her research interests include numerical methods in electromagnetism, microwave radiometry, antenna analysis, and design. Currently, her research is mainly related to the study of numerical methods applied to sea surface emissivity and their characterization at L-band and the MIRAS/SMOS project.

Dr. Vall-llossera, along with the other member of the radiometry group at UPC, was awarded the "Primer Premio Ciutat de Barcelona d'Investigació Tecnològica" in 2001, the "Primer Premio Duran Farell de Investigación Tecnológica" in 2002, the "Premi Nacional de Telecomunicacions 2003," and the "Premi Salvà i Campillo 2004."

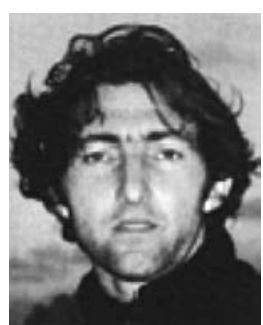

Nicolas Reul received the B.S. degree in marine sciences engineering from Toulon University, La Garde Cedex, France, in 1993 and the Ph.D. degree in physics (fluid mechanics) from the University AixMarseille II, Marseille, France, in 1998.

From 1999 to 2001, he was a Postdoctoral Researcher with the Applied Marine Physics Department, team of Prof. M. Donelan, Rosenstiel School of Marine and Atmospheric Science, University of Miami, Coral Gables, FL. Since 2001, he has been a Permanent Researcher with the Département d'Océanographie Physique et Spatiale, Institut Français de Recherche pour l'Exploitation de la Mer, Plouzané, France, responsible for the activities concerning the future SMOS satellite mission. The focus of his research program is to improve understanding of the physical processes at the air-sea interface and passive/active remote sensing of the ocean surface. He has experience in applied mathematics, physical oceanography, electromagnetic wave theory, and its application to ocean remote sensing. He is currently a member of the ESA/SMOS Science Advisory Group. 